\title{
Growing of the Inhomogeneities and Acceleration Phase in a Universe with $\Lambda$ Decay
}

\author{
M. de Campos \\ Physics Departament - Universidade Federal de Roraima, \\ Av. Ene Garcez 2413, Campus do Paricarãna, Bloco III, \\ Bairro Aeroporto, Boa Vista, Roraima, 69304-000, Brazil \\ Physics Departament - Universidade Federal Fluminense, Av. Gal. Milton Tavares de Souza, \\ s/n - Campus da Praia Vermelha, CEP 24210-346, Niterói - RJ, Brazil
}

Received on 4 August, 2007

\begin{abstract}
We study a model with decay of dark energy and creation of the dark matter particles. We integrate the field equations and find the transition redshift where the evolution process of the universe change from decelerated to an accelerated phase expansion, and briefly discuss the transition epoch and the broken of the strong energy condition. Finally, we examine the growing of the inhomogeneities.
\end{abstract}

Keywords: Dark matter; Dark energy; Growing of the inhomogeneities; Acceleration of the universe

\section{INTRODUCTION}

Before the results from supernova of the type IA observations appear in the literature, that indicates an accelerated expansion of the universe, L. Krauss and M. Turner have called our attention that "The Cosmological Constant is Back ". They cited the age of the universe, the formation of large scale structure and the matter content of the universe as the data that indicates the insertion of cosmological constant [1]. The cosmic microwave background radiation anisotropy and large scale structure, also indicates this acceleration process of the universe [2], [3],[4], [5].

The mechanism that triggered the acceleration of the universe has not been identified, and the simplest explanation for this process is the inclusion of a non null cosmological constant. However, the inclusion of cosmological constant creates new problems. Some of them are old, as the discrepancy among the observed value for the energy density of the vacuum and the large value suggested by the particle physics models [6], [7]. In spite of the problems caused by the inclusion of $\Lambda$, the cosmological scenario with $\Lambda$ has a good agreement in respect to the age of the universe estimate, the anisotropy of the microwave background radiation and the supernova experiments. Beyond this, making several assumptions concerning with the spectrum of fluctuations in the early universe and the formation of the galaxies, G. Efstathiou suggests that the small value of cosmological constant can be explained by the anthropic principle [8].

The evidence that these new components of the universe, dark matter and dark energy, are different substances has been considered in literature [9]. Generally the dark matter component is considered as weakly interacting massive particles and the dark energy component is associated to some form of a scalar field. A link between both components to a scalar field is studied by Padmanabhan and Choudury [10]. Consequently, both components have a scale-dependent state equation, Although, today, both components are unknown is respect to the your nature.

An alternative model that furnish a negative pressure in the cosmic fluid and results in an accelerated expansion of the universe is known as open system cosmology [11] and other alternative dark energy models have been reviewed in [12]. In this model the particles number in the universe do not conserve and the energy-momentum tensor is reinterpreted in the Einstein's equations, where appear an extra pressure known as creation pressure and is negative [13],[14]. The creation process is due to the expenses of the gravitational field and is an irreversible process. One of the attractive features of the hypothesis of particle production is the relation among the large scale properties of the universe and the atomic phenomena [15].

So, in the present, there is significant observational evidence that we live in an accelerated universe and a lot of alternative explanations for the cosmic acceleration have appeared in the literature [16], [17].

In this work we consider a different rate for diluting of the material components due to decaying of the dark energy component as the same way that the authors considered in [18] and study the transition of the decelerated expansion for an accelerated expansion of the universe and the relation with the broken of strong energy condition. Several aspects of the of the approach by Wang and Meng [18] are investigate in [19]

In other words we have creation of dark matter particles at the expenses of the $\Lambda$-decay. Finally, we look at the growing for the density contrast and call attention that to obtain the solution for the density contrast that appear in the work [18] the authors must consider a dependence for the $\Lambda$-decay that is discarded by current constraints on the dark energy equation of state.

\section{THE BASIC EQUATIONS OF THE MODEL}

Only a few years after introducing the field equations of the general relativity theory, Einstein proposed adding a cosmological constant to the original equations which assumes the form

$$
R_{\alpha \beta}-\frac{1}{2} g_{\alpha \beta} R-\Lambda g_{\alpha \beta}=8 \pi G T_{\alpha \beta}
$$

Originally, the cosmological constant was put in the right side of Einstein equations, consequently interpreted as an incre- 
ment in the geometric side. However, other option is to include $\Lambda$ in the left side of the Einstein equations and consider it as a different form of energy, with density and pressure characteristics. Beyond this, $\Lambda$ can be consider as a time function.

In this work the space-time is considered as homogeneous and isotropic, characterized by FRW metric

$$
d s^{2}=d t^{2}-R(t)^{2}\left(d r^{2}+r^{2} d \theta^{2}+r^{2} \sin \theta^{2} d \phi^{2}\right),
$$

and the energy momentum-tensor is the usual perfect fluid,

$$
T_{\mu v}=\left(\rho_{d m}+\rho_{\Lambda}+P\right) u_{\mu} u_{v}-P g_{\mu v} .
$$

$P$ is the pressure, $\rho_{\Lambda}=\frac{\Lambda}{8 \pi G}$ is the dark energy density, $\rho_{d m}$ is the dark matter density, while $u_{\mu}$ is the four velocity. So, taking into account that the reference system is just the matter filling it, the Einstein field equations can be written as:

$$
\begin{aligned}
\frac{\ddot{R}}{R} & =-\frac{1}{6} 8 \pi G\left(\rho_{d m}+\rho_{\Lambda}+3 P\right), \\
\frac{\dot{R}^{2}}{R^{2}} & =\frac{1}{3} 8 \pi G\left(\rho_{d m}+\rho_{\Lambda}\right),
\end{aligned}
$$

Based in the inflation predictions and in the data from the WMAP [20], we assume spatial flatness.

Assuming a coupling between vacuum energy and material components of the universe, with creation of matter particles, we can write the conservation law

$$
u_{\mu} T_{; v}^{\mu \nu}=-u_{\mu}\left(\frac{\Lambda g^{\mu \nu}}{\kappa}\right) ; v
$$

that in the present study assumes the form

$$
\dot{\rho}+3 \frac{\dot{R}}{R} \rho=-\dot{\rho}_{\Lambda},
$$

since that, although the vacuum is decaying the state equation for the vacuum remain with the usual aspect $P_{\Lambda}=-\rho_{\Lambda}$ and $\rho=\rho_{d m}+\rho_{\Lambda}$. The development of $\Lambda$ decaying models can be looked at [21], [22], and the thermodynamic behaviour at [23].

In the absence of dark energy decay we have $\rho_{\dot{d} m}+$ $3 \frac{\dot{R}}{R} \rho_{d m}=0$, which integration results $\rho_{d m}=\rho_{d m 0} R^{-3}$, where the subscript 0 refers to the present time and we consider that the present value for the scale factor is normalized $\left(R_{0}=1\right)$.

On the other hand, if the dark energy decays into cold dark particles, we can assume that the energy density will dilute in a different rate, namely

$$
\rho_{d m}=\rho_{d m 0} R^{\varepsilon-3},
$$

where the positive constant $\varepsilon$ furnish the deviation from the standard case without creation.

Rewriting Eq.(5) as

$$
\frac{d \rho_{d m}}{d R}+3 \frac{\rho_{d m}}{R}=-\frac{d \rho_{\Lambda}}{d R},
$$

and integrating, results

$$
\rho_{\Lambda}=\rho_{\Lambda 0}-\frac{\varepsilon}{3-\varepsilon} \rho_{d m 0}\left\{1-R^{\varepsilon-3}\right\} .
$$

With auxilious of Eqs. (4), (6) and (8) we find

$$
\dot{R}^{2}-K_{I} R^{2}-K_{I I} R^{\varepsilon-1}=0,
$$

where $K_{I}=\frac{8 \pi G}{3}\left\{\rho_{\Lambda 0}-\frac{\varepsilon}{3-\varepsilon} \rho_{d m 0}\right\}$ and $K_{I I}=\frac{8 \pi G}{3-\varepsilon} \rho_{d m 0}$, which integration results

$$
R(t)=\left(\frac{K_{I I}}{K_{I}}\right)^{\frac{1}{3-\varepsilon}}\left\{\sinh \sqrt{K_{I}}(3-\varepsilon) t / 2\right\}^{\frac{2}{3-\varepsilon}}
$$

With the scale factor (10) we can calculate the Hubble function $(H=\dot{R} / R)$ and the deceleration parameter $\left(q=-\frac{\ddot{R} R}{\dot{R}^{2}}\right)$, respectively:

$$
\begin{aligned}
H(t) & =\sqrt{K_{I}} \frac{\cosh \left\{(3-\varepsilon) \sqrt{K_{I}} t / 2\right\}}{\sinh \left\{(3-\varepsilon) \sqrt{K_{I}} t / 2\right\}} \\
q(t) & =-1+\frac{3-\varepsilon}{2}\left[\cosh \left\{(3-\varepsilon) \sqrt{K_{I}} t / 2\right\}\right]^{-2} .
\end{aligned}
$$

The analysis of $156 \mathrm{SNe}$ realized by Riess et al. [2] point out to the present acceleration at $99.2 \%$ level and for the past deceleration $(q>0)$ beyond the redshift $Z_{t}=0.46 \pm 0.13$ at $99.8 \%$ level, where the subscript $t$ refers to the transition point which the universe change the signal for the deceleration parameter. With auxilious of Eqs. (10) and (12) the redshift of the transition can be calculated and put in terms of the age of the universe $\left(t_{0}\right)$, namely

$$
Z_{t}=\left\{\left(\frac{1-\varepsilon}{2}\right)^{-1 / 2} \sinh \left[(3-\varepsilon) \sqrt{K_{I}} t_{0} / 2\right]\right\}^{\frac{2}{3-\varepsilon}}-1,
$$

We can use this relation and the expression for the Hubble function to write the present Hubble function in terms of transition redshift $\left(Z_{t}\right)$, namely

$$
H_{0}=\left\{K_{I}+\frac{2 K_{I}}{(1-\varepsilon)\left(1+Z_{t}\right)^{3-\varepsilon}}\right\}^{1 / 2}
$$

Note that, when more recent is the transition for the acceleration process we obtain an younger universe.

On the other hand, the acceleration expansion of the universe is not possible if the strong energy condition holds. Since that we have a transition of the acceleration to deceleration expansion process, naturally the strong energy condition must be broken at the identical redshit which the transition occurs.

Then, we have:

$$
\rho_{d m}+\rho_{\Lambda}+3 P=-\frac{3}{\kappa}\left\{2 K_{I}+(\varepsilon-1) K_{I I} R^{\varepsilon-3}\right\} .
$$

So, from (15) we find that the strong energy condition is broken for

$$
R_{\text {sec }}=\left\{\frac{(1-\varepsilon) K_{I I}}{2 K_{I}}\right\}^{\frac{1}{\varepsilon-3}} .
$$

Note that, the redshift correspondent to the above scale factor is

$$
Z_{s e c}=\left[\frac{K_{I I}(1-\varepsilon)}{2 K_{I}}\right]^{\frac{1}{\varepsilon-3}}-1
$$


that is identical to the redshift of the transition point.

To finish, we want to make some commentaries about the growing of the inhomogeneities . The equation for density contrast in the presence of $\Lambda$-term is given by [24]

$$
R^{2} \frac{d^{2} \delta}{d R^{2}}+\frac{3}{2}\left(1+\frac{\Lambda}{3 H^{2}}\right) R \frac{d \delta}{d R}+\left(\frac{\Lambda}{2 H^{2}}-\frac{3}{2}\right) \delta=0 .
$$

Considering the decay of $\Lambda$ into particles the equation for $\delta$ assumes the form:

$$
R^{2} \frac{d^{2} \delta}{d R^{2}}+\frac{3+\varepsilon}{2} R \frac{d \delta}{d R}+\frac{\varepsilon-3}{2} \delta=0
$$

which integration results

$$
\delta \propto R^{\frac{\sqrt{\varepsilon^{2}-6 \varepsilon+25}-\varepsilon-1}{4}} .
$$

This expression is identical to the obtained by Wang and Meng. However, to obtain the differential equation (19), we use the equations (4), (6) and (8). In addition we must consider that the formation of the inhomogeneities occurs at some time ago such that $R_{\delta}<<R_{0}=1$, where $R_{\delta}$ is the scale factor at the born of the inhomogeneities. Beyond this, the vacuum density today must a very small value. With this approximation and help of equations (4), (6) and (8), we find $\Lambda=\varepsilon H^{2}$. This kind of dependence for $\Lambda$ is inconsistent with the current constraints on the dark energy equation of state according the authors of [18] and [25].

When the $\Lambda$ terms are ignored in eq. (18) we obtain the usual solution $\delta \propto R$, that evolves faster then the solution given by (20).
[1] M. L. Krauss and M. Turner, Gen. Relativ. Gravit., 27, 1137 (1996).

[2] A. G. Riess et al., Astron. J., 116, 1009 (1998).

[3] A. G. Riess et al., Astrophys. J., 607, 665 (2004).

[4] S. Perlmutter et al., Astrophys. J., 517, 565 (1999).

[5] D. N. Spergel, Astrophys. J. Suppl. Ser., 170, 377 (2007).

[6] S. Weinberg Rev. Mod. Phys., 61, 1009 (1989).

[7] J. Garrig and A. Vilenkin, hep-th/00011262, (2000).

[8] G. Efstathiou, Mon. Not. R. Astron. Soc, 274, L73 (1995).

[9] H. Sandvik et al., Phy. Rev. D, 69, 123524 (2004).

[10] T. Padmanabhan and T. R. Choudury, Phy. Rev. D, 51, 290 (1937).

[11] I. Prigogine, J. Geheniau, E. Gunzig, and P. Nardone, Gen. Relativ. Gravit., 21, 767 (1989).

[12] J. A. Lima, Braz. J. Phys., 34, 194 (2004).

[13] J. S. Alcaniz and J. A. Lima, Astron. Astrophys. J, 349, 729 (1999).
[14] J. A. Lima and J. S. Alcaniz, Astron. Astrophys. J, 348, 1 (1999).

[15] W. Mac Crea, Proc. R. Soc. London A, 206, 562 (1951).

[16] S. Carrol, Astro-ph/0310342, (2003).

[17] J. Peebles and B. Ratra, Rev. Mod. Phys., 750, 559 (2003).

[18] P. Wang and X. Meng, Class. Quant. Grav., 22, 283 (2005).

[19] J. S. Alcaniz and J. A. S.Lima, Phy. Rev. D, 72, 063516 (2005)

[20] D. N. Spergel, Astrophys. J. Suppl. Ser., 148, 175 (2003).

[21] J. C. Carvalho, J. A. Lima, and I. Waga, Phy. Rev. D, 46, 2404 (1992).

[22] J. A. Lima and J. M. F. Maia, Phy. Rev. D, 49, 5597 (1994).

[23] J. A. S. Lima, Phy. Rev. D, 54, 2571 (1996).

[24] A. Vale and J. Lemos, Mon. Non. R. Astron. Soc., 325, 1197 (2001).

[25] S. D. H. Hsu, Phys. Lett., B 594, 13 (2004). 